\title{
To Assess the Fibular Grafting in Gap Nonunion and Bony Defects Created by Trauma or Tumour Excision
}

\section{Rajendra Kumar Shakunt, Brijesh Sharma, Sushil Kumar Saini, Sabeel Ahmad, Ashwani Sadana and CP Pal*}

Department of Orthopaedics, S N Medical College, India

*Corresponding Author: CP Pal, Department of Orthopaedics, S N Medical College, Agra, India.
Received: August 10, 2021

Published: September 06, 2021

(C) All rights are reserved by CP Pal., et al.

\begin{abstract}
Introduction: Treating a large bone defects is really challenging for orthopaedician. The bone defect created by tumour excision, bone loss as sequelae to infection and trauma. Various methods popularly cancellous Bone graft, free fibular graft, and bone transport with the help of LRS (Limb Reconstruction System) and external fixator technique used to fill the defect. Fibula is very common bone to use for filling a defect created after bone resection either after tumor or in bony nonunion. The treatment of these bone gaps has evolved significantly during the past two decades. This study aims to assess the role of fibular grafting (nonvascularized) in gap non-union and bony defect created after tumour resection as a treatment modality in terms of financial burden, hospital stay as well as technically demanding.

Method: This was a prospective study conducted at S.N Medical College, Agra from April 2018 to May 2021. In this period of three years we analysed hospital records to find out the cases treated by fibular strut grafts. Among 30 cases 20 were taken those fulfilling the inclusion criteria, in which there were 15 tumours and 5 post traumatic shortening.

Results: This study had 65\% (13/20) stable, pain free limb movements, resumed over a period of one year without any assisted device. 7 patients who did not gain normal functions, in which 3 cases had fair result, and 4 got failed.
\end{abstract}

Conclusion: Overall experiences with non-vascularized fibular graft for reconstructing bony defects are promising, more handy and universal.

Keywords: Fibula; Graft; Nonunion; Tumour; Bone

\section{Introduction}

Treating a large bone defects is really challenging for orthopaedician. The bone defect created by tumour excision, bone loss as sequelae to infection and trauma [1-4]. Among various method and technique fibula Grafting is a popular method for bridging the gap in bone defects. Fibula is very common bone to use for filling a defect created after bone resection either after tumor or in bony nonunion [5]. Various methods popularly cancellous Bone graft, free fibular graft, and bone transport with the help of LRS (Limb Reconstruction System) and external fixator technique used to fill the defects [6]. Bone transport has been one of the best way to treat complicated post-traumatic bone loss but during this procedure many problems are anticipated like axial rotation, anatomical mal-alignment, fixator in situ for prolonged period, longer hospital time and associated antecedent mental or psychological problems. Fibular strut graft used in all age group patients. Fibular grafting is easier and short harvesting time with avoid economic burden over 
the poor patients in developing countries and small setups. There is several methods came in existence to treating the bone defect since last 2 years. There is recent surge of bone graft after tumor resection, bone gap in atrophic nonunion of fracture site and augmentation in revision arthroplasties in last few years due to less comorbidities. As we knew vascularized fibula may provide rapid biological incorporation, more growth potential in skeletally immature patients, and around compromised soft tissue. This procedure might not be possible at some of the centers because lack of technical expertise, however non-vascularized fibular graft in long term follow up patients has shown promising results. Hence, we decided to reconstruct the bony gaps after tumour excision and in atrophic nonunion with the help of non-vascularized fibular graft [7].

\section{Aim of the Study}

This study aims to assess the Role of fibular grafting in gap nonunion and bony defect to study the role of fibular grafting in gap non-union as a treatment modality in terms of financial burden, hospital stay as well as technically demanding.

\section{Materials and Methods}

This was conducted at S.N Medical College, Agra from April 2018 to May 2021, and a prospective study. Patients were selected from patients attending Emergency Department and OPD of Orthopaedics and fulfilling the inclusion criteria. Written informed consent was taken to every patient. Inclusion criteria of this study was those patients with Gap union due to trauma and tumor, Gap non-union sequelae to post infection, Post-surgical gap non-union, Compound fractures with bone loss. Excluded patients were-infected gap non-union as it may be result of osteomyelitis. Over a period of 3 years we looked in the patients records to find out cases, those were treated by fibular graft. Among 30 cases, 20 were taken those fulfilling the inclusion criteria, in which there were 15 tumours and 5 post traumatic shortening. Age varies between 10 years to 55 years; among then 6 were females and 14 males.

\section{Initial management of patients}

Preoperative evaluation done for the tumour cases were complete haemogram (CBC), serum studies, appropriate radiographs and skeletal survey. Serum studies consisted serum calcium, phosphorus and ALP. CT and MRI of involved part were also done including nearby joints. With the help of MRI and CT we came to know the extent of lesion, neurovascular involvement and cortical integrity etc. Based on these investigations, wide resection planned along with reconstructions.

On the basis of HPE (histopathological examination) report we decided the extent of excision of tumours. HPE was done as excisional biopsy to know the nature of tumour like benign or malignant. These patients are only selected for surgeries if the tumour is localized and not involved the neurovascular structures with the help of imaging modalities. Other investigations are also done to get surgical clearance. All patients were given single dose of antibiotics before surgery and intraoperative one more shot if surgery is prolonged. We used two teams in such kind of surgeries to preserve precious time, one to resect tumour and other prepare the graft.

\section{Operative procedure}

Under anesthesia, incision was made taking the biopsy scar in same line. Tumour excised with adequate margins which is around 2 to $5 \mathrm{~cm}$. while excising the tumour care has taken to not contaminate the healthy tissue. Size of the resected segment was taken to measure the graft length (Shown in figure 1). Through posterolateral approach skin incision was made depending upon graft length. If we plan to resect the proximal $1 / 3^{\text {rd }}$ of fibula common peronial nerve to be protected by identifying its course at biceps tendon. Subperiosteal striping was done carefully at proximal fibula. Fibula resected according to the defects. According to above technique proximal fibula harvested in 5 cases for GCT (giant cell tumour) of DER (distal end radius) and shaft of fibula for rest cases. To promote union, rigid immobilization and internal fixation was done with hardware like plate with screws or $\mathrm{k}$ wire.

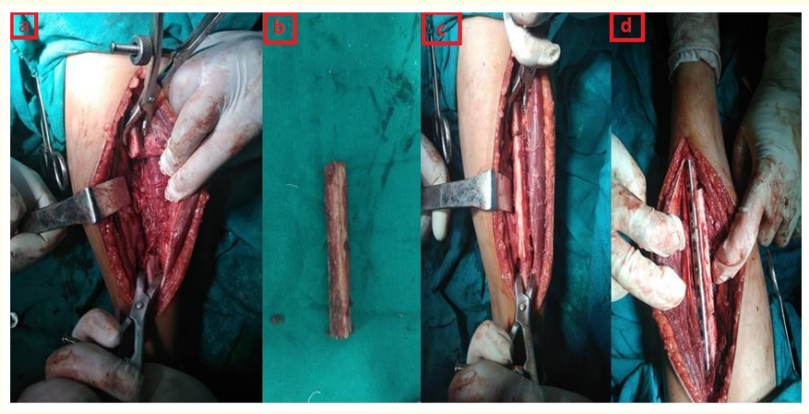

Figure 1: (a) intraoperative pictures showing fibula harvesting, (b) fibular strut graft, (c, d) showing non-vascularized fibular grafting at recipient site which is being fixed with plate and screws. 


\section{Post-operative protocols}

All patients received antibiotics over 3 post op days. Suture removed at $14^{\text {th }}$ days of surgery simultaneously checking the suture line, and patients sent home with plaster cast for 8 weeks. After 8 weeks gradual ROM at the adjacent joint started under supervision. In lower limb toe touch mobilization started at 3 months and in upper limb partial ROM exercises at 8 weeks. All patients were followed up to 6 month (once in month) and undergone for clinical and radiological evaluations. After that 2 monthly up to one year (mostly union happened in this period).

We routinely checked our patients for graft incorporation, recurrence and functional evaluation. Criteria for union were presence of smooth external continuity and radiolucency at graft and host junction. The oncological evaluation was done by looking patient survival, recurrence and metastasis. Functional evaluation was done by Mankin., et al. scoring system [8].

\section{Results}

Over a period of three years we analysed the hospital records to find out the cases treated by fibular strut grafts. Among 30 cases 20 were taken those fulfilling the inclusion criteria, in which there were 15 tumours and 5 post traumatic shortening. In this study upper limb and lower limb shares 50\% - 50\% cases. Humerus and distal radius put together constitute $50 \%$ of the cases. The age of the patients ranged from 10 years to 55 years. The average age taken is 28 years. Out of 20 cases 6 were females and rest males. Graft incorporation was assessed radiographically. Graft union time in 17 out of 20 patients between 3 to 10 months period. Average time of graft incorporation was 6.5 months. 3 months in children, 5 months in case of wrist and 6 to 10 months in case of other bones. In the remaining 3 cases the graft did not incorporate. Oncological evaluation was done for tumours conditions, among 15 tumour cases 14 doing well and one got recurrence of GCT. Results were based on functional outcome (Table 1).

\begin{tabular}{|l|c|}
\hline Outcome & Total no. of cases \\
\hline Excellent & 10 cases \\
\hline Good & 3 cases \\
\hline Fair & 3 cases \\
\hline Failed & 4 cases \\
\hline
\end{tabular}

Table 1: Results were based on functional outcome which was analysed according to Mankin., et al. criteria.

\section{Discussion}

Goal of this study to cure disease and make patient self-dependent, improve his/her daily activities of living with functional limb. Thus our aim was complete removal of pathology with adequate margins in $1^{\text {st }}$ setting itself, that's why en-bloc resection was done for aggressive/recurrent benign lesion. Reconstruction is mandatory after removal of tumorous lesion to preserve the function and normal anatomy. Other methods of reconstructions were also considered like autograft, allograft, and prosthetic replacement. Use of allograft is bit risky due to incompatibility; need preservation for long duration, selection of suitable donor and other many formalities which are really cumbersome, though it shows promising results. Surgeons must anticipate all the risk factor related to the graft applications like infections, rejections and delayed healing. Graft can be auto or allograft which can be further divided in to vascularized and non-vascularized. Vascularized fibular autograft is demanding and required technical expertise like microscopic surgeons. Non-vascularized fibular graft incorporation as an autograft is more quick and compatible than an allograft [9]. Moreover, it is easily approachable and minimal donor site morbidity [5]. However this is easy and can be performed by most of orthopaedician in average set up without disturbing the biological equilibrium at donor site.

In this study non-vascularized fibular graft were used for filling defects in humerus, proximal tibia, distal radius, femur, that araised due to resection of tumours and defects created by trauma (Table 2). We had total 15 case of tumour, among them 14 cases of benign and one case of malignant, which were treated with fibular grafting. Among 15 tumour, 5 cases were GCT involving DER (distal end radius) which was reconstructed with the proximal fibula, providing structural similarity (Figure 2) except one case of recurrence. Manish Chadha., et al. [10] reported good result in 8 patients out of 9 patients with giant cell tumors involving distal end radius treated with non-vascularized fibular graft.

Another 5 cases, the defect were around knee joint (proximal and distal femur and proximal tibia tibia). Sometime the fibular graft augmented with bone cement or graft, even hardware used to fix the graft (Figure 3). After clearing the disease around joints maintaining the anatomical alignment some time leads to joint stiffness. That's why functional outcome was good around large joints. Kuriakku P Dominic., et al. [11] also reported same result in twelve cases treated with fibular grafting augmented either 


\begin{tabular}{|l|c|c|}
\hline \multirow{4}{*}{ Trauma } & Humerus & 2 \\
\cline { 2 - 3 } & Tibia & 1 \\
\cline { 2 - 3 } & Femur & 2 \\
\hline \multirow{4}{*}{ Tumour } & Humerus & 3 \\
\cline { 2 - 3 } & Femur & 2 \\
\cline { 2 - 3 } & Proximal Tibia & 3 \\
\cline { 2 - 3 } & Distal End Radius & 5 \\
\cline { 2 - 3 } & Distal Tibia & 2 \\
\hline
\end{tabular}

Table 2: This table shows total number of cases (20) including trauma and tumour involving the bone.

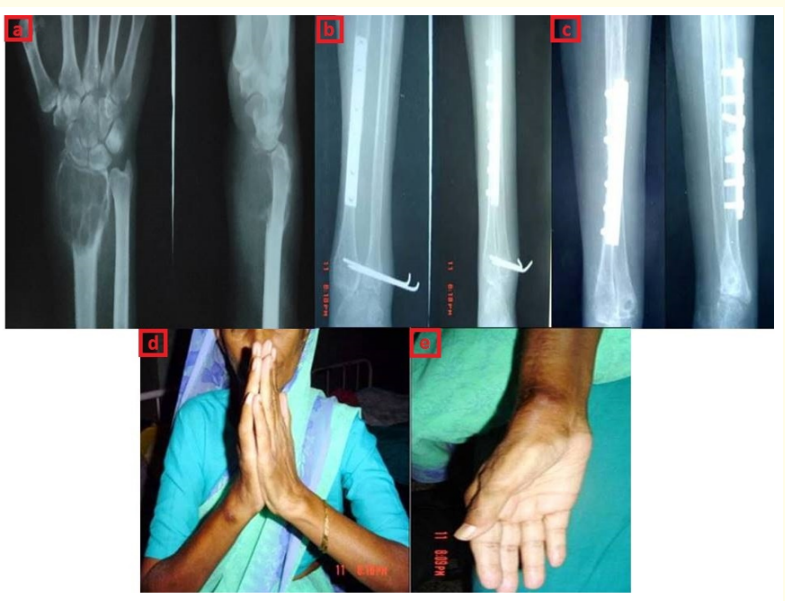

Figure 2: (a) This is 46 years old lady having giant cell tumour at distal end radius of right forearm, (b) post op day 2 showing proximal $1 / 3^{\text {rd }}$ of fibula fixed with plate and screws at the resected end of distal end radius, (c) incorporation of fibular graft with radius having radiolucency at fixation site after 6 months, (d, e) A good range of motion at wrist in clinical picture after 6 months.

with cement or bone grafting. After Distal tibia GCT resection, reconstruction was done by arthrodesis of tibia and calcaneus with hardware. Here the functional was not good due to calcaneal related problems like pain and deformity. Yener Saglik., et al. [12] also had successful result in two case of distal tibia giant cell tumor treated by a non-vascular fibular graft after tumour resection and arthrodesis of tibia and calcaneus.

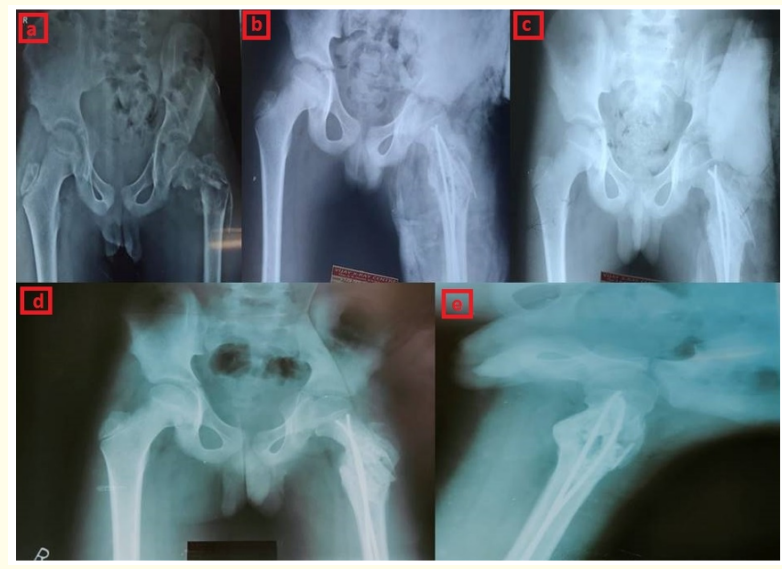

Figure 3: (a) This is X-ray of 11 years old child, having aneurysmal bone cyst in the proximal femur with pathological fracture (at basi-cervial and inter-trochantric region), (b) post op day 2 , which shows fracture fixed with rush nail along with strut fibula graft, (c) 3 months after the procedure anteroposterior $\mathrm{X}$ ray of hip and proximal femur, $(\mathrm{d}, \mathrm{e})$ at 6 months of follow up $\mathrm{X}$ rays shows excellent union and remodeling at pathological fracture site without reoccurrence of tumor.

Among trauma group we had 5 cases, out of 5, 3 got failed. Out of 3 cases, 2 had compound injuries and initially treated elsewhere with ex-fixator. Both the cases presented with compromised soft tissue locally which could not heal due to extensive procedure resulting in wound dehiscence and infection. They managed by the LRS and ilizarov fixators. Failure in the $3^{\text {rd }}$ case is most probably due to inadequate fixation of graft even after adequate time that was subsequently managed by bone grafting with plate application. This study correlate with the result of the study done by Iyengar., et al [13].

This study had 65\% (13/20) stable, pain free limb movements resumed over a period of one year without any assisted device (Figure 4). 7 patients who did not gain normal functions, in which 3 cases had fair result, and 4 got failed. Those 3 fair outcome patients were because of persistent pain, in which two patients with dis- 
tal femur reconstruction had joint arthritis and deformity at knee, and last one with distal tibia reconstruction had ankle stiffness and hind foot deformity.

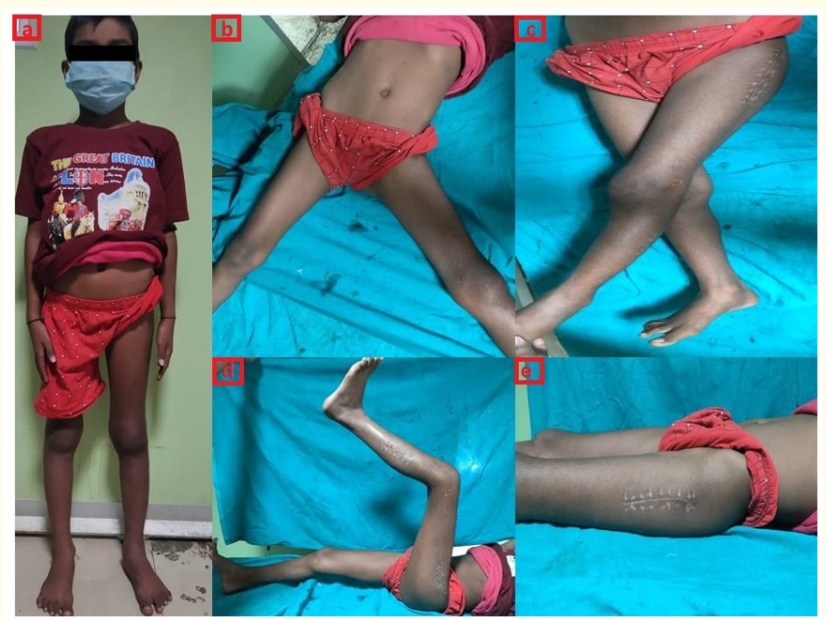

Figure 4: (a) Clinical pictures of Same child at 6 months (Shown in figure 3), (b, c, d) showing 40 - 50 degree of abduction, 30 - 40 degree of adduction and 100 degree of flexion $(d, e)$ showing fibular graft site without any surgical site complications, no flexion deformity seen.

4 patients with failure were due to infections, nonunion and recurrence of disease. Similar results were achieved by Al-Zahrani., et al. [14] who had primary union in 92\% patients treated by nonvasculerized fibular graft augmented with corticocancellous graft along its whole length, Indications were varied and included infection, fracture with bone loss, nonunion, bone tumours, bone cyst and pseudoarthrosis.

\section{Conclusion}

After bone resection the created defect was successfully reconstructed by fibular graft - Which gives pleasing results in term of function and cosmetics. Most of the cases present very late in traumatic bone defects with soft tissue compromise, in those results are not satisfactory. However these patients are managed well if they seek early treatment. According to our view, non-vascularized fibular grafts in bony defect reconstructions are promising. As we knew this is short term study and need further evaluation and some more inputs with new innovations.

\section{Conflict of Interest}

Author declares that there is no financial or any conflict of interest exists.

\section{Bibliography}

1. Al-Zahrani S., et al. "Free fibular graft still has a place in the treatment of bone defects". Injury 24 (1993): 551-554.

2. Enneking WF., et al. "Autogenous cortical bone grafts in the reconstruction of segmental skeletal defects". Journal of Bone and Joint Surgery American 62 (1980): 1039-1058.

3. Yadav SS. "Dual fibular grafting for massive bone gaps in the lower extremity". Journal of Bone and Joint Surgery American 72 (1990): 486-494.

4. Tuli SM. "Bridging of bone defects by massive bone gaps in tumorous conditions and in osteomyelitis". Clinical Orthopaedics 87 (1972): 60-73.

5. Ortiz-Cruz E., et al. "The results of transplantation of intercalary allografts after resection of tumors. A long-term follow-up study". Journal of Bone and Joint Surgery American 79 (1997): 97-106.

6. Cattaneo R., et al. "The treatment of infected nonunions and segmental defects of the tibia by the methods of Ilizarov". Clinical Orthopaedics and Related Research 280 (1992): 143-152.

7. Jobe TM. “Micro surgery: Campbell's Operation Orthopaedics. $10^{\text {th }}$ edition., Philadelphia, PA: Mosby 4 (1998): 3335.

8. Partick J. “Getty: Complications and functional outcomes of reconstruction with an osteoarticular allograft after intra articular resection of the proximal aspect of the humerus". Journal of Bone and Joint Surgery 81 (1999): 8.

9. Dhammi IK., et al. "Giant cell tumours of lower end of the radius problems and solutions". The Indian Journal of Orthopaedics 39 (2005): 201-205.

10. Chadha M., et al. "Autogenous non-vascularized fibula for treatment of giant cell tumor of distal end radius". Archives of Orthopaedic and Trauma Surgery 130 (2010): 1467-1473.

11. Kuriakku Puthur Dominic., et al. "Extended curettage and reconstruction with proximal fibula for treating giant cell tumor 
of lateral femoral condyle: A prospective study". Archives of Clinical and Experimental Surgery 6 (2017): 189-194.

12. Saglik Y., et al. "The Use of Fibular Autograft and Ankle Arthrodesis for Aggressive Giant Cell Tumor in the Distal Tibia: A Case Report". Foot and Ankle International 29 (2008): 438-441.

13. Iyengar. "Reconstruction of Traumatic, Open Supracondylar Femoral Fractures by Autologous Fibular Strut Grafting and Cortico-Cancellous bone grafting". Journal of Orthopaedic Trauma 32 (2018): 75-81.

14. Al-zahrani S., et al. "Free fibular graft still has a place in the treatment of bone defects". Injury 24 (1993): 551-554.

Volume 4 Issue 10 October 2021

(C) All rights are reserved by $\mathrm{CP}$ Pal., et al. 